\title{
Time-consuming eyelashes: an audit of trichiasis management
}

This article was published in the following Dove Press journal:

Clinical Audit

I3 July 2010

Number of times this article has been viewed

\section{Mona Khandwala \\ Sarju Dey \\ Muddassar Hussain \\ Carole A Jones}

Maidstone and Tunbridge Wells NHS Trust, Maidstone, UK
Correspondence: Mona Khandwala Maidstone and Tunbridge Wells NHS Trust, Hermitage Lane, Maidstone MEI6 9QQ, UK

$\mathrm{Tel}+44794 \quad 1604630$

Fax +44I62 2226255

Email mona.khandwala@gmail.com
Purpose: To assess the efficacy, appropriateness, and efficiency of treatment for trichiasis in the ophthalmic department of a UK district general hospital.

Methods: Fifty-nine patients with trichiasis were treated between September 2005 and September 2006. A case note review identified the cause of trichiasis and classified patients into those with lid malposition $(n=10)$ and those with lash distortion $(n=44)$. We audited for effective treatment (relief of symptoms which allowed patient discharge), appropriate treatment ( $\leq 3$ epilations before treatment review, 1-5 aberrant lashes electrolysed, $>5$ aberrant lashes treated by cryotherapy, all patients with lid malposition referred to the oculoplastic clinic, and listed for surgery), and efficient treatment ( $<3$ epilations and $<4$ electrolysis treatments or a single operation prior to discharge).

Results: For patients with lid malposition, $80 \%$ were discharged after one operation, $80 \%$ were listed appropriately, and $87.5 \%$ patients were efficiently treated. For those with lash distortion, the success rate was 54\% with epilation alone and with electrolysis, whilst success with cryotherapy was $78 \%$. Prior to electrolysis, on average a patient attended 10 times for epilation. Cryotherapy was utilized after an average of three electrolysis treatments. The average number of patient visits for epilation was 5 (range 1-26). The average number of patient visits for electrolysis was 2 (range 1-6).

Conclusion: Patients with lid malposition were managed well. Epilation alone was undertaken inappropriately, resulting in inefficient treatment and unnecessary clinic visits. To improve patient care, a treatment algorithm is now displayed in all clinic rooms.

Keywords: eyelash, removal, epilation, electrolysis, cryotherapy

\section{Introduction}

Trichiasis is a generalized term used to describe a range of eyelash abnormalities which result in contact between lashes and the ocular surface. It is a common and distressing condition which can be difficult to treat. Appropriate diagnosis and management prevents ocular damage, provides symptomatic relief, and avoids frequent and unnecessary hospital visits.

Symptomatic trichiasis has been treated with epilation, electrolysis, cryotherapy, and lid surgery. Electrolysis can destroy lash follicles but requires careful treatment of individual lash roots. It has been reported to be successful for a small number of lashes, but can cause lid scarring if treatment is heavily and repeatedly applied. ${ }^{1}$ Cryotherapy is effective, with temperatures up to $-20^{\circ} \mathrm{C}$ being required. Electrolysis, laser ablation, electrosurgery, and single lash cryotherapy have all been used to treat the aberrant eyelash with a success rate of about $70 \%$, although the rate increases with repetitive treatment. ${ }^{2}$ 
Table I Numbers of patients classified to each group

\begin{tabular}{ll}
\hline Lid abnormality & \\
\hline Cicatricial/involutional entropion & 10 \\
Border entropion & 5 \\
Ocular cicatricial pemphigoid & 5 \\
Lash abnormality & \\
Primary & 27 \\
Secondary & 12 \\
\hline
\end{tabular}

The aim of this audit is to evaluate the management, specifically the efficacy, appropriateness, and efficiency of treatment for trichiasis in the ophthalmic department of our hospital. Maidstone Hospital is a general hospital serving a population of approximately 750,000 . The eye department provides casualty, general, and subspecialty ophthalmic clinics, some of which are nurse-led.

\section{Patients and methods}

Trichiasis is a common problem encountered in ophthalmic departments, but is often dealt with in a superficial way by less qualified and inexperienced staff. Our experience has been that this leads to an unnecessarily large number of return visits but rarely stimulates insightful research, as is demonstrated in the paucity of published data.

There are currently no standardized national guidelines or protocols for the treatment of trichiasis. A literature review failed to reveal any randomized controlled trials comparing treatment options. Various studies have analysed these treatments individually with variable success rates. There is no consensus on an optimum treatment protocol, and hence we have established our own standards to compare with current hospital practice.

\section{Standards}

Effective treatment is defined as a patient discharge with relief of symptoms. Appropriate treatment is defined as: $\leq 3$ epilations before moving onto the next treatment modality. If $1-5$ aberrant lashes are noted, they are to undergo electrolysis. ${ }^{2}$ However, if the patient has $>5$ aberrant lashes, they are to be treated by cryotherapy. ${ }^{3}$ Finally, all patients with lid malposition are to be referred to the oculoplastic clinic, and listed for surgery. ${ }^{1}$ Efficient treatment is defined as $<4$ electrolysis treatments ${ }^{4}$ and $<3$ epilations.

The above standards were based on published papers on individual trichiasis management modalities. Kezirian ${ }^{2}$ and Tucker $^{3}$ recommend epilation or electrolysis for a localized area of trichiasis with $<5$ lashes involved. For more extensive trichiasis, ie, $>5$ lashes, they recommend cryotherapy or surgery. Khooshabeh ${ }^{1}$ suggests that aggressive use of cryotherapy or electrolysis in cases with lid malposition can induce further scarring and shrinkage of the tarsus, resulting in frank cicatricial entropion, and thus recommends reconstructive surgery to rebuild a stable eyelid.

\section{Patient demographics}

The case notes of all patients who attended the Nurse-led Lid Clinic (NLLC) between September 2005 and September 2006 were reviewed. Data were collected on patient demographics (age, gender), etiology of trichiasis, ocular comorbidity, treatment (epilation, electrolysis, cryotherapy, and surgery), number of clinic attendances, and final outcome.

\section{Diagnosis}

We used the classification of causes of trichiasis suggested by Barber and Dabbs. ${ }^{5}$ The etiology of trichiasis is shown in Table 1. Patients with lid abnormality included nine with lower-lid involutional entropion and one with upper-lid cicatricial entropion. Border entropion ${ }^{5}$ is defined as a small degree of entropion characterized by conjunctivalization of the meibomian gland orifices and anterior placement of the mucocutaneous border of the lid. All five patients with border entropion were on long-term glaucoma medication, two were on beta-blockers, two on prostaglandin analogs, and one on betamethasone and atropine eye drops for rubeotic glaucoma. The secondary lash abnormality group had isolated misdirected lashes secondary to meibomitis. However, in 27/39 cases, no cause for the misdirection could be found (primary lash abnormality). There were no patients with distichiasis.

The five patients with ocular cicatricial pemphigoid were excluded from further analysis because they attended the external diseases clinic and were managed separately. For the purpose of this analysis, the border entropion group are included with the lash abnormality patients because they followed the same treatment pathway.

The tenets of the Helsinki Declaration were followed. Ethical Committee approval was not required for this audit.

\section{Outcome measures}

Efficacy and appropriateness of treatment were analysed for both patient groups and for all treatment modalities; in addition, efficiency of treatment was analysed for epilation and electrolysis.

\section{Results}

Sixty-eight patients were identified as having attended the NLLC for trichiasis between September 2005 and September 
2006. We included 59 patients in the final analysis because five had died and four sets of notes could not be obtained. There were 34 males and 25 females of mean age 79 (45-98) years. Most patients were referred by their GP $(n=46)$, followed by 12 optician referrals and one selfreferral. At the patients' first visit, they were seen by staff of various grades. Twenty patients were seen by a senior ophthalmologist, seven by a junior ophthalmologist, and 20 were seen initially by a nurse, and then referred on to the NLLC for review.

\section{Group I: Lid abnormality (excluding border entropion)}

Surgery was the principal form of treatment for the lid malposition group and was found to be both effective and appropriate. Surgery was performed in $7 / 10$ patients and, in six cases $(85.7 \%)$, surgery was successful after the first operation (lower-lid entropion repair), whilst one patient required three operations for cicatricial upper-lid entropion. Eight patients were listed appropriately (80\%), although one refused surgery. Two cases were inappropriately treated by epilation and electrolysis.

\section{Group 2: Lash abnormality (including border entropion)}

Patients with lash root distortion and those on long-term topical medication were treated by epilation alone, epilation followed by electrolysis, or a combination of epilation, electrolysis, and cryotherapy.

Success with epilation alone was achieved in 6/11 patients (54\%) and with electrolysis in 13/24 (54\%) patients. Nine patients who had undergone multiple epilation and electrolysis treatments were referred for cryotherapy from the NLLC, after a senior medical opinion was sought. Of the nine patients who underwent cryotherapy, success was achieved in seven patients (78\%), all of whom needed only one treatment. The remaining two patients are awaiting their second cryotherapy treatment (Figure 1).

Appropriate treatment was defined as $\leq 3$ epilation treatments and $\leq 4$ electrolysis sessions for $1-5$ lashes. On average, patients underwent epilation 10 times before electrolysis was performed. Cryotherapy was utilized after an average of three electrolysis treatments. All patients who underwent cryotherapy were appropriately treated (Figure 2).

Epilation was inefficient, being performed $<3$ times in only $1 / 11(9 \%)$ patients. The average number of patient visits before discharge was five (range 1-26).

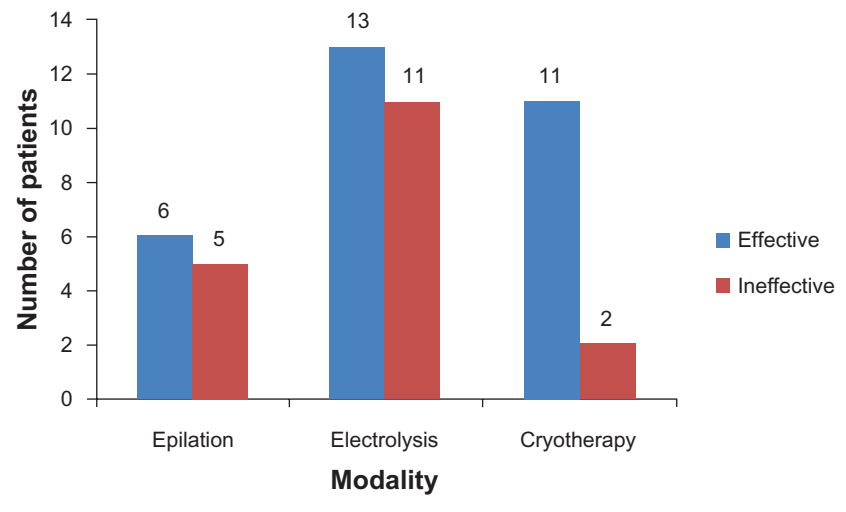

Figure I Efficacy of treatment (lash distortion group).

Electrolysis was efficient, performed less $<4$ times in $21 / 24(87.5 \%)$ patients, with an average of two visits (range 1-6, Figure 3)

The overall results of treatment for the patient cohort were efficient treatment in $61 \%$ of cases, appropriate treatment in $72 \%$, and effective treatment in $37 \%$.

\section{Discussion}

Trichiasis is a difficult condition to manage and often requires specialist oculoplastic surgical management. This audit showed that the current treatment of trichiasis in the department was effective and appropriate, especially in those with lid malposition (surgical management). The electrolysis treatment in the lash distortion group was effective and appropriate. However, the use of epilation was both inappropriate and inefficient. The patients attended multiple times before epilation treatment was effective, with a range of $1-26$ visits to the NLLC. Patients were undergoing excessive epilation and consequently attending too frequently. This could be attributed to seeing junior or inexperienced staff at their first attendance or the lack of a formal treatment protocol.

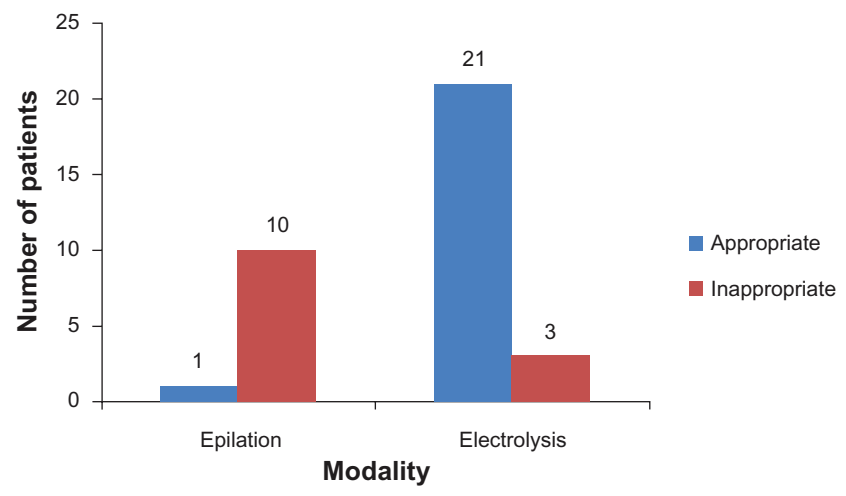

Figure 2 Appropriateness of treatment (lash distortion group). 


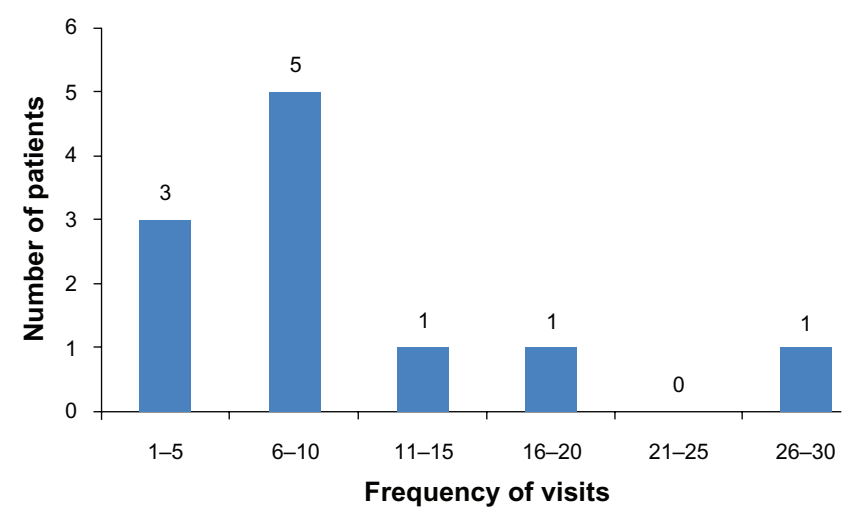

Figure 3 Efficiency of epilation.

Our treatment efficacy is comparable with that reported in the literature. Kezirian ${ }^{2}$ quotes a $56 \%$ success rate with electrolysis, whilst our audit showed a $54 \%$ success with this modality. Possible complications of electrolysis include lid scarring, but no complications were found in this study. The success of cryotherapy (78\%) recorded in this audit compared favorably with that reported by Delaney et al at $56 \%-90 \%{ }^{6}$ and Collin et al at $84 \% .{ }^{7}$ However, in the presence of cicatricial disease, the success rate with cryotherapy decreased to $40 \%$ at one year and $34 \%$ at four years. ${ }^{8}$ In trichiasis due to trachoma, Reacher et $\mathrm{al}^{9}$ reported a success rate of $27 \%-56 \%$. Cryotherapy-related complications include meibomian gland dysfunction, skin depigmentation, and recurrence, but in this small study no complications were identified. Figueiredo et $\mathrm{al}^{10}$ reported on a series of 216 cases with trichiasis. They subdivided them into two groups, ie, those with pure trichiasis (no eyelid deformity) and those related to entropion. Electrocautery of follicles of aberrant eyelashes had a recurrence rate of $41 \%$, which is comparable with our findings. They suggest that small areas (about $3 \mathrm{~mm}$ ) could be treated with destruction of follicles

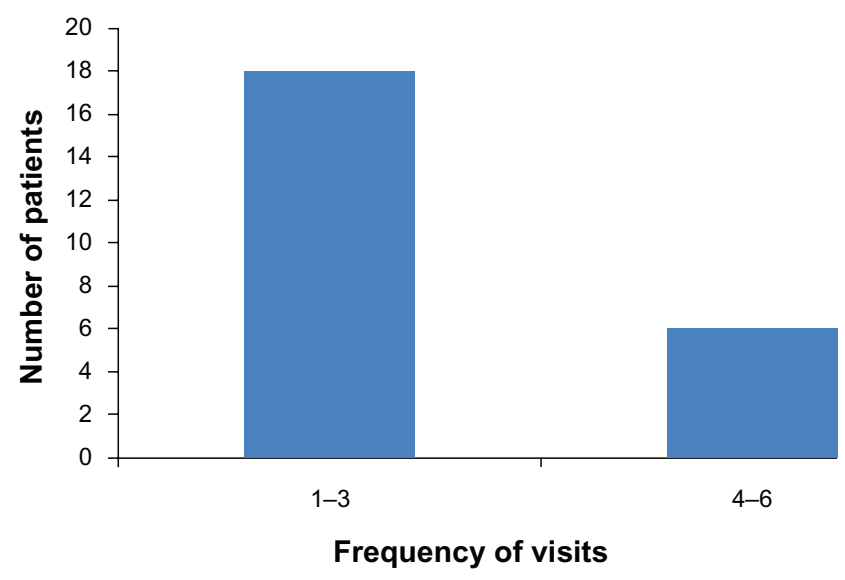

Figure 4 Efficiency of electrolysis. using electrocautery or cryotherapy, and larger areas could be treated by intermarginal separation with cutaneous-mucosal or tarso-conjunctival grafting.

Two recent studies have published success rates for surgical treatment of entropion with trichiasis. Mills et al ${ }^{11}$ reported on "central lower eyelid thinning with trichiasis" as a unique subset of elderly patients with entropion. Of their patients, 20/41 were refractory to initial treatment that included lubrication, epilation, and/or electrolysis and went on to have surgery. Of these, $16 / 20(80 \%)$ were deemed successful. Bleyen et al ${ }^{12}$ report an $85 \%$ overall success rate in their series on 126 eyelids with cicatricial entropion or trichiasis who underwent the Weis procedure. Fourteen percent developed a recurrence and required a second procedure. Our surgical success rates are similar to these studies, with an $87.5 \%$ success rate.

There is no specific mention in the literature regarding efficiency of various modalities of treatment for trichiasis, and the paucity of studies in the literature on trichiasis treatment preclude an evidence-based setting of standards.

We have established our own standards based on the success rates of various studies with different treatment modalities. Although studies in the literature have reported varying success rates with multiple modalities, there are few studies with an overview of the management of the aberrant eyelash. Because efficiency of treatment has financial implications, improved efficiency would benefit both patients and hospital departments. With implementation of recommendations, it is envisaged that the diagnosis and management of this condition in the department will improve. We are aware that this data collection was a retrospective case note review and thus limited information could be obtained from the notes.

\section{Conclusion}

The current treatment regime for patients with trichiasis is effective and appropriate when compared with set standards. The number of epilation treatments was inappropriately high and thus inefficient. In order to improve the efficiency and appropriateness of the management of trichiasis in our hospital, we have designed a treatment algorithm which is presented here in the form of a flow chart (Figure 5). This has been put in all our patient and casualty clinic rooms to assist the attending doctor or nurse. We hope that this will lead to more appropriate treatment plans for patients with trichiasis. We aim to reaudit in 12 months to close the audit loop after the new treatment protocol has been implemented. 
Treatment algorithm for trichiasis

Eye, Ear and Mouth Unit Maidstone Hospital

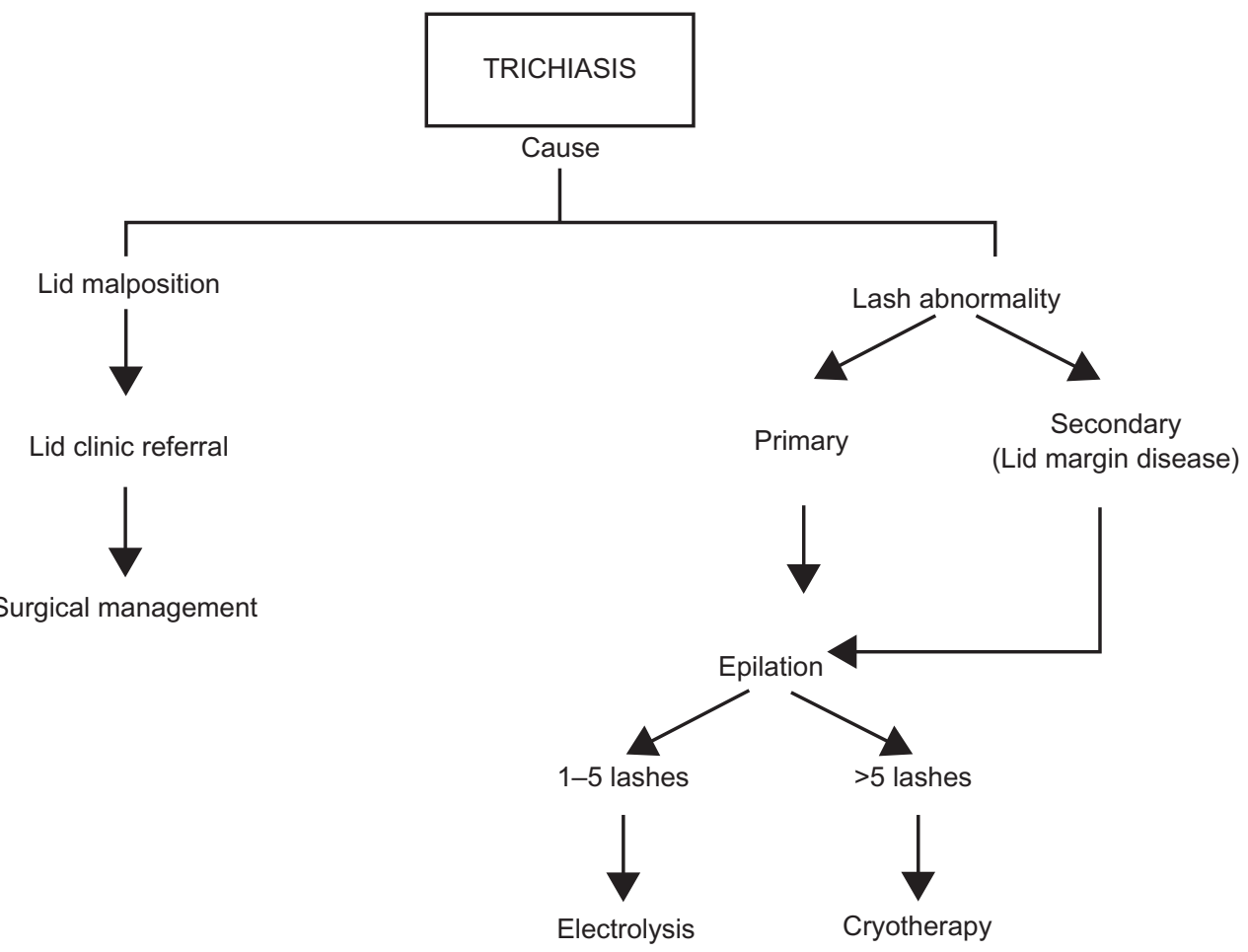

Figure 5 Trichiasis treatment algorithm.

\section{Acknowledgment}

This audit was presented as a poster at the Association of Vision Research (ARVO) annual meeting at Fort Lauderdale, Florida, in 2008.

\section{Disclosure}

None of the authors have any financial interests to declare. There was no funding for the audit.

\section{References}

1. Khooshabeh R. The Unwanted Eyelash. The Royal College of Ophthalmologists of London. 2002;24:21.

2. Kezirian GM. Treatment of localized trichiasis with radiosurgery. Ophthal Plast Reconstr Surg. 1993;9(4):260-266.

3. Tucker SM. Management of trichiasis - What works and what doesn't. Techniques in Ophthalmology. 2003;1(3):168-172.

4. Elder MJ. Anatomy and physiology of eyelash follicles: Relevance to the lash ablation procedures. Ophthal Plast Reconstr Surg. 1997;13:21-25.

Clinical Audit

\section{Publish your work in this journal}

Clinical Audit is an international, peer-reviewed, open access journal focusing on the processes and outcomes of clinical audit in any area of healthcare. All aspects of patient care are addressed within the journal and practitioners from all disciplines are invited to submit their work. Areas covered include: Publication of audits; How an audit has changed practice;
5. Barber K, Dabbs T. Morphologic observations on patients with presumed trichiasis. Br J Ophthalmol. 1988;72:17-22.

6. Delaney MR, Rogers PA. A simplified technique for trichiasis and distichiasis. Austr J Ophthalmol. 1984;12:163-166.

7. Johnson RC, Collin JR. Treatment of trichiasis with a lid cryoprobe. Br J Ophthalmol. 1985;69:267-270.

8. Elder M, Bernauer W. Cryotherapy for trichiasis in ocular cicatricial pemphigoid. Br J Ophthalmol. 1994;78:769-771.

9. Reacher MH, Munoz B, Alghassany A, Daar AS, Elbualy M, Taylor HR. A controlled trial of surgery for trachomatous trichiasis of the upper lid. Arch Ophthalmol. 1992;110:667-674.

10. Figueiredo AR, Soares EJ. Trichiasis: Diagnosis and management. Long term follow-up of 216 cases. Orbit. 1992;11(3):137-146.

11. Mills DM, Meyer DR. Central lower eyelid thinning with trichiasis: Characterization and management of a unique subset of entropion in elderly patients. Ophthal Plast Reconstr Surg. 2009;25:445-449.

12. Bleyen I, Dolman DJ. The Weis procedure for management of trichiasis or cicatricial entropion of either upper or lower eyelids. Br J Ophthalmol. 2009;93:1612-1615.
Practical tips on how to do audits and to avoid pitfalls; How audits have changed patient care; Calls and justifications for new audits. The manuscript management system is completely online and includes a very quick and fair peer-review system, which is all easy to use. Visit http://www.dovepress. com/testimonials.php to read real quotes from published authors. 